\title{
ESTUDO DA TEORIA DAS FILAS APLICADO EM CASA LOTÉRICA NA CIDADE DE CATAGUASES/MG
}

\author{
Bruno Lacerda Marques: lacerdabrr@gmail.com \\ Isabela Thais Ferreira Santiago: isabela.fsantiago@outlook.com.br \\ Larissa Bastos Machado: larissabm@outlook.com.br \\ Thais da Silva Brizi: tata_brizi@hotmail.com \\ Tiago Bittencourt Nazaré: tiago@unis.edu.br
}

\begin{abstract}
Resumo: O presente artigo descreve e analisa o estudo com relação à teoria de filas aplicado a uma casa lotérica situada na cidade de Cataguases-MG. Tal abordagem se justifica por contribuir com o aprofundamento do tema, uma vez que, filas são consideradas um grande problema para a população. O estudo se justifica por ser um serviço oferecido a um tempo considerável no Brasil, mas que vem sofrendo alterações ao longo do caminho para atender melhor os clientes e consequentemente gerar a satisfação dos mesmos. Com intuito de avaliar e posteriormente definir como funcionam as filas de uma lotérica, foi realizado métodos de análise sobre as teorias das filas. Utilizou-se uma abordagem qualitativa e quantitativa. O estudo foi feito através de conhecimentos de pesquisa operacional, para elaboração do estudo, foi analisado o tempo de atendimento dos caixas e o intervalo que os clientes formam à fila, com intuito de conhecer os processos de gerenciamento e controle dos operadores de caixa para um bom atendimento.
\end{abstract}

Palavras-chave: Pesquisa Operacional. Teoria das Filas. Casa Lotérica.

\section{Introdução}

No Brasil, a primeira loteria de que se tem notícia foi construída em 1784, em Vila Rica (atualmente, Ouro Preto), antiga capital de Minas Gerais. Com o dinheiro arrecadado foram construídos os prédios da Câmara dos Vereadores e da Cadeia Pública. A prática foi adotada em todo país, sendo que o governo fornecia concessões para sua exploração preferencialmente às Santas Casas, aos orfanatos e aos hospitais, e também a particulares. Mais tarde, em 1962 foi definido que as lotéricas seriam administradas pela Caixa Econômica Federal, em um decreto assinado pelo presidente João Goulart. (APARECIDA, 2007).

Para contextualizar, lotérica é a unidade que comercializa todas as loterias federais, os produtos assemelhados e atua na prestação de todos os serviços delegados pela Caixa Econômica Federal. É importante ressaltar que o serviço oferecido pela Casa Lotérica oferece diversos serviços de atendimento, tais como: pagamento de faturas, abertura e depósitos bancários, apostas, item este muito utilizado pela população em busca de uma realização. Nesse contexto, para atendimento destes serviços surgem as filas, requisitando uma plausível atenção.

De acordo com Prado (1999), o estudo das filas iniciou-se em 1908 com uma abordagem matemática. A partir do surgimento do computador na década de 50, a modelagem de fila pôde ser examinada através da representação, que consiste em processos que permite o alcance de conclusões sobre o comportamento de um sistema, com a tentativa de imitar o funcionamento do sistema real por meio de um modelo.

O estudo da Teoria das Filas contribui para a manutenção, gerenciamento e melhorias no processo de atendimento, indagando evitar desperdícios e gargalos e satisfazendo ambas as partes. A Teoria das Filas é um dos tópicos da Pesquisa Operacional com aplicações no campo da administração das empresas, tratando de problemas de congestionamento de sistemas, na qual, a principal característica é a presença de clientes solicitando serviços de alguma 
forma (ANDRADE, 2004).

A finalidade do presente artigo é realizar um estudo que verifique o sistema e processos de uma Casa Lotérica. A agência escolhida para análise se localiza em Minas Gerais, no município de Cataguases, no Bairro Centro. Está no mercado há 4 anos, possui 4 guichês de atendimento e 5 funcionários, contando com o gerente do estabelecimento.

\section{Referencial Teórico}

Para Lovelock e Wright (2002), uma fila é a representação de uma linha de pessoas, veículos, objetos físicos ou intangíveis que aguardam sua vez de serem atendidos. O processo de formação de filas ocorre quando o número de chegadas excede à capacidade do sistema de atendimento e geralmente está relacionado a problemas relativos à administração da capacidade. Portanto fila é uma sequência de pessoas, produtos ou serviços, aguardando por atendimento, ou sequencia do processo. Consiste do processo de chegada e distribuição do tempo de serviço, do número de servidores e da capacidade do sistema.

Normalmente, a fila resulta da falta deliberada ou não, de programação, pois, se fosse possível organizar as chegadas e os serviços, seria possível também evitar a espera dos clientes e consequentemente não haveria fila. Na maioria dos casos, porém, é impossível programar as filas, embora não desejadas, são inevitáveis.

Uma fila ocorre sempre que a procura por um determinado serviço é maior que a capacidade do sistema de prover este serviço. Em determinados dias, há um maior fluxo de pessoas procurando o mesmo tipo de atendimento, desta forma, é comum ocorrer um acumulo de demanda. Para tratar desse problema, quando se observa o fluxo de clientes, por exemplo, é possível perceber que estes não chegam de forma contínua durante todo o dia, mas, há estágios de maior e menor concentração no período, gerando filas e em outros momentos, estrutura ociosa.

Para complementar Fogliatti e Mattos (2007) afirmam que o estudo das filas é uma previsão do comportamento das mesmas com o propósito de regular a infraestrutura e o número de equipamentos, insumos e recursos tecnológicos necessários para evitar problemas por falta de recursos nesta fila de forma a manter os clientes satisfeitos.

A Teoria de Filas é de extrema importância no ato de estudar as características do processo, pois possibilitará a redução dos problemas causados aos clientes, como por exemplo, atrasos, fazendo com que aconteça a fidelização dos clientes e que a empresa forneça um serviço de qualidade. (Oliveira, 2006)

O estudo baseado desta teoria é realizado através de análises e propriedades medíveis das filas que facilitam a verificação do comportamento de sistemas reais em situação aleatória. Desta forma, é possível dimensionar a capacidade de atendimento ao se combinar custo do serviço, qualidade oferecida e investimentos nos gargalos (Oliveira, 2006).

A teoria parte do seguinte pressuposto a formação de filas excede a capacidade de fornecer determinado serviço e envolve o estudo matemático utilizando ferramentas de tratamento estatístico ou estocástico. (Dávalos, 2012)

\section{Componentes do Sistema de Filas}

Os personagens de uma fila são o servidor e o cliente, como apresentado na FIGURA 1, e os clientes são originados por uma fonte, pela população. Ao chegarem a um estabelecimento 
de serviço podem iniciar o serviço imediatamente ou esperar em uma fila, caso o guichê de atendimento de serviço esteja ocupado. Quando se conclui um serviço, chama-se de forma automática outro cliente, que já se encontra à espera na fila, sendo ele o referido próximo que está no aguardo. Caso a fila esteja vazia, aguarda-se a chegada de um novo cliente.

Figura 1. Processo de Fila

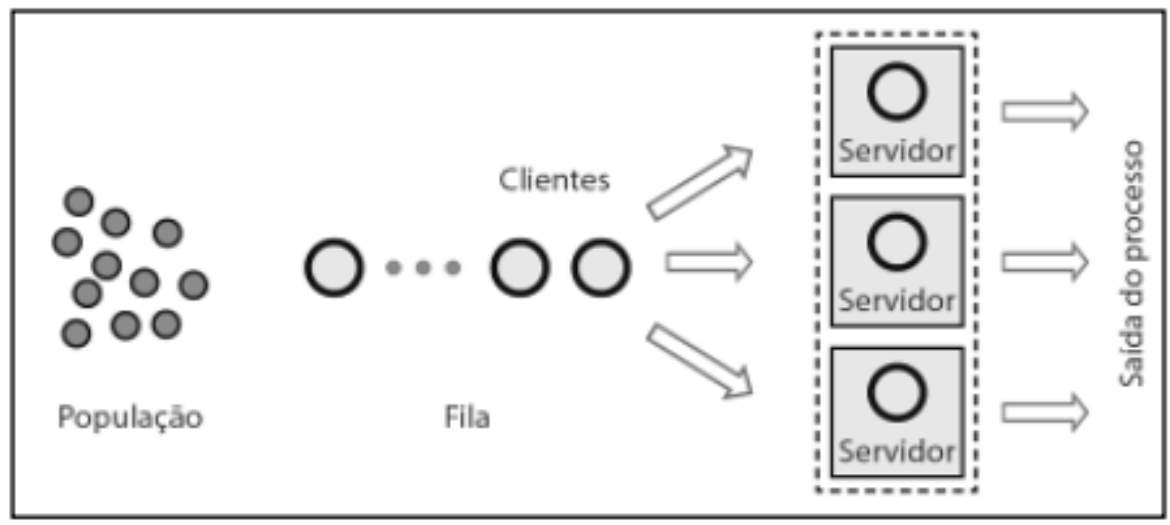

Fonte: Marques (2001)

Na figura 1, mostra-se o processo de fila de um determinado estabelecimento, no qual se fundamenta a fila de uma lotérica. Nela, apontam-se os personagens do processo: O cliente, originado pela descrição população, e o servidores, pessoas que realizam o atendimento dos serviços do estabelecimento. A população (cliente) gera a fila de atendimento, e espera pelo servidor. Após o atendimento concluído, é diretamente encaminhada para a saída do processo, para que o próximo seja atendido.

Segundo Gross (2008) e Camelo (2010), existem seis características básicas que compõem o processo de filas: processo de chegada de clientes, padrões de serviços prestados, disciplina da fila, capacidade do sistema, número de canais de atendimento e estágios do serviço.

As filas são caracterizadas por sua extensão e quantidade. Sendo a primeira caracterizada como limitada ou ilimitada. De acordo com Heizer e Render (2001) a fila se caracteriza limitada quando por lei ou alguma restrição física existente, impede de crescer infinitamente, e se caracteriza ilimitada quando não existe nenhuma restrição de crescimento.

Quanto à quantidade, o número de filas se define por únicas ou múltiplas. Sendo fila única aquela em que todos os clientes aguardam na mesma fila sem nenhuma divisão e múltipla aquela em que as filas se dividem em dois ou mais servidores e/ou filas que convergem a um ponto central para redistribuir-se (Davis, 2001).

\section{Características de Chegada}

Segundo Davis (2001), há quatro princípios que relatam a chegada em um processo, sendo elas: padrão de chegada, que é definido tanto como controlável, quanto como incontroláveis, contagem dos clientes, baseada em cada entrada podendo ser definida como unitária, quando uma singularidade representando o número menor trabalhando no procedimento ou um múltiplo de chegada. As filas geralmente contam com um índice de chegada ou uma média 
dos clientes por intervalo de tempo. Além do grau de tolerância que é o tempo em que o cliente permanece na fila esperando, e o grau de impaciência que é quando o cliente avalia a fila para decidir se espera ou não pelo atendimento.

\subsection{Relatório das Filas}

O condicionamento da fila abrange um agrupamento de regras determinantes para ordenamento do atendimento dos clientes que compõe a fila. A quantidade de cliente, ampliação da fila, período médio de espera, e a eficácia do serviço prestado, são fatores que influenciam a alternativa das diretrizes de prioridade (Jacobs e Chase, 2012).

Dessa forma Ritzman e Krajwsk (2004), ressaltam que na maioria dos procedimentos de atendimento é utilizada a regra por de ordem de chegada, ou seja, quem chega primeiro é atendido primeiro (PEPS), sendo assim o cliente que está no primeiro lugar na fila possui maior prioridade, quanto ao último que chegou. Entretanto, existem outros tipos de diretrizes como data mais aproximada (DEMP) ou período de processo mais curto (TPEP).

\subsection{Saída do Sistema}

Dado o fim do atendimento do cliente, o mesmo poderá escolher entre duas formas de saída possível, como: retomar a fila e candidatar-se a um novo atendimento e/ou pode existir uma forma de retrabalho. (Davis, 2001).

\section{Metodologia}

A pesquisa realizada na Casa Lotérica da Cidade de Cataguases é denominada em qualitativa e quantitativa. Uma pesquisa quantitativa é aquela que tudo pode ser quantizado buscando a relação causa-efeito do fenômeno analisado, quando a qualitativa não necessita de uso de dados quantizados, mas sim da análise de relação do mundo real. O estudo foi desenvolvido através dos conhecimentos de pesquisa operacional sobre teoria das filas.

Tanto a abordagem quantitativa, quanto à qualitativa, se aproxima, dentro de suas diferenças e especificidades, e são utilizadas como base de apoio para a análise de dados. Os métodos quantitativos supõem uma população de objetos de observação comparável entre si e os métodos qualitativos enfatizam as especificidades de um fenômeno em termos de suas origens e de sua razão de ser. (Ibid, p. 63 apud. Ferreira, 2015).

É de extrema importância que o pesquisador, avalie o campo em que seu trabalho está situado, além dos limites e potencial dos métodos quantitativos e qualitativos.

Segundo Dal-Farra e Lopes:

\footnotetext{
“(...) os estudos quantitativos e qualitativos possuem, separadamente, aplicações muito profícuas e limitações deveras conhecidas, por parte de quem os utiliza há longo tempo. Por esta razão, a construção de estudos com métodos mistos pode proporcionar pesquisas de grande relevância, desde que fique simples de identificar com clareza as potencialidades e as limitações no momento de aplicar os métodos em questão."
}

Para a análise do tempo foi utilizado cronometro de mão, para evitar possíveis erros. A coleta de dados foi realizada nos dias 01/11/2018 e 05/11/2018, das 12h00min ás 13h00min, 
totalizando 60 amostras. Por meio de um benchmarking com o proprietário da Casa Lotérica, foi identificado que neste período se concentra um maior fluxo de atendimento por conta da data que os trabalhadores recebem seus pagamentos. Desta forma a pesquisa foi feita em 2 dias aleatórios por 1 hora, para se obter um melhor resultado.

\section{Descrição do Sistema}

A loteria estudada tem duas filas sendo uma preferencial para idosos e apostas de jogos e outra sendo fila única com o atendimento por ordem de chegada. As duas filas são caracterizada como FIFO (o primeiro a entrar é o primeiro a sair). Na casa lotérica estudada há quatro guichês, operados por atendentes, que realizam serviços de pagamentos, apostas de jogos, entre outros.

\section{Identificação do Empreendimento Pesquisado}

A casa lotérica estudada está situada no Bairro Centro, próxima à Estação de Trem da cidade de Cataguases, atuando como representante da Caixa Econômica Federal. A mesma atua ofertando diversos tipos de serviços, como pagamentos, abertura de contas além de jogos da Loteria Federal. A loteria tem cinco funcionários fixos sendo quatro compostos por mulheres, as atendentes, e um composto por um homem, o gerente, que trabalham em regime de 44 horas semanais, sendo 8 horas diárias, com intervalo de almoço de 2 horas. Os horários de atendimento são de segunda a sexta e com um turno de trabalho de 4 horas aos sábados. Foi identificado com o estudo, que os serviços desta lotérica alcançam aproximadamente 450 atendimentos por dia, pondendo chegar a $1250 \mathrm{em}$ dias onde o fluxo for maior. As informações utlizadas no presente artigo foram cedidas pelo gerente, concedendo assim a devida autorização para serem usadas como base de pesquisa.

\section{Resultado}

Para realização do estudo verificou-se o histórico da empresa, suas características, e quais as principais limitações em seu processo produtivo. E através dos dados coletados foi realizado o estudo dos dados com base na teoria das filas.

\section{Dados Coletados}

Após coleta dos dados foi utilizado o editor de planilhas e cálculos (Excel), para dar tratamento aos mesmos e verificar como estava o tempo de atendimento da fila em relação ao tempo de chegada dos clientes, o critério adotado foi de exclusão de 95\%. Abaixo, interpretado por gráficos, observa-se a linha limite de dispersão representada pela linha de cor (vermelha), sendo que tal linha é o percentil de $95 \%$.

Desta forma, no Gráfico 1 foi excluído os 3 pontos acima da linha que possuíam os seguintes valores: $3,82-5,25-4,87$ minutos. 


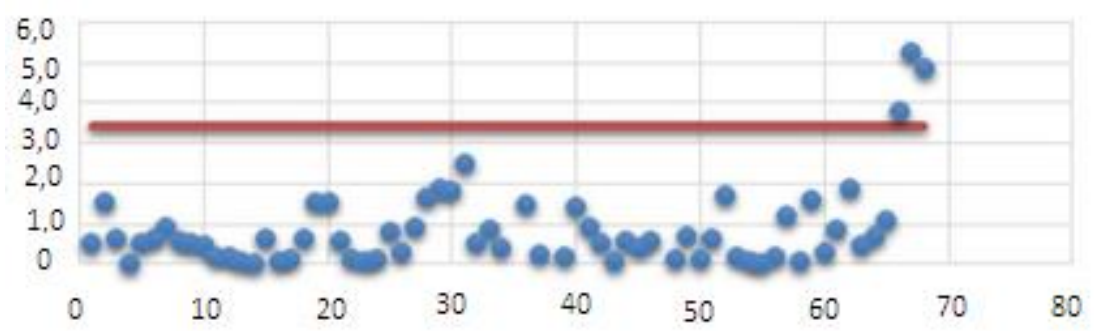

Fonte: http://www.abepro.org.br/biblioteca/tn_stp_231_350_29698.pdf

E no Gráfico 2, os 3 pontos que estavam acima da linha continham os seguintes valores: 5,57 $-5,78-6,18$ minutos. Nos gráficos 1 e 2 antes da exclusão dos dados que estão acima do limite estipulado, pode-se ressaltar que todos os elementos que estão abaixo da linha (reta) estão dentro da média de dispersão e que devem ser excluídos, todos os que estão acima.

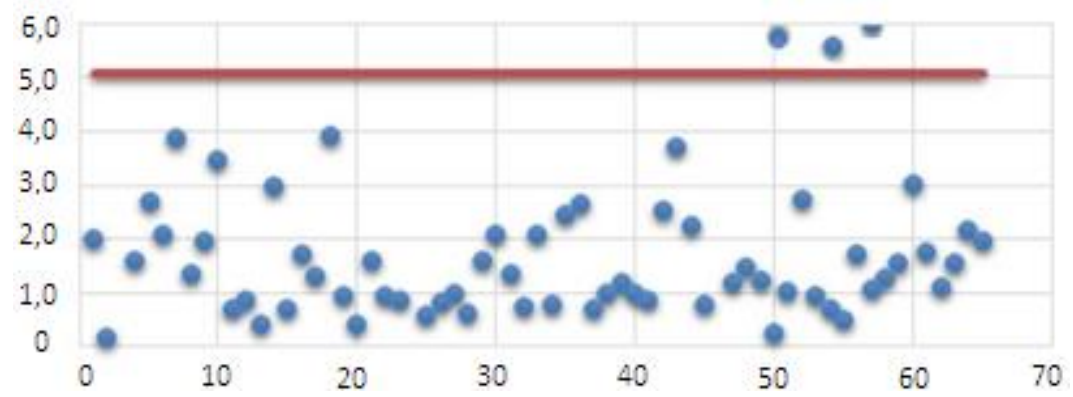

Fonte: http://www.abepro.org.br/biblioteca/tn_stp_231_350_29698.pdf

Após a exclusão dos pontos mencionados foram criadas as tabelas 1 e 2 de frequência, referente à mensuração da chegada, atendimento, e classificação de intervalos.

Ao verificar a Tabela 1 o intervalo entre as classes é de 0,52 minutos, pode-se observar onde o valor mínimo de todos os elementos analisados em relação à chegada é de 0,02 minutos e o máximo de 2,50 minutos, onde o maior intervalo de frequência é o número 1 $(0,02-0,52)$ com uma taxa de frequência de $44 \%$. 
Tabela 1 - Intervalo de Chegada

\begin{tabular}{|c|c|c|c|c|}
\hline INTERVALO E & INTERVALO D & FREQ. ABS. & $\begin{array}{c}\text { FREQ. } \\
\text { RELATIVA }\end{array}$ & $\begin{array}{c}\text { FREQ. } \\
\text { ACUMULADA }\end{array}$ \\
\hline 0,52 & 1,01 & 21 & $34 \%$ & $77 \%$ \\
\hline 1,01 & 1,51 & 4 & $6 \%$ & $84 \%$ \\
\hline 0,02 & 0,52 & 27 & $44 \%$ & $44 \%$ \\
\hline 1,51 & 2,01 & 9 & $15 \%$ & $98 \%$ \\
\hline 2,01 & 2,50 & 1 & $2 \%$ & $100 \%$ \\
\hline
\end{tabular}

Fonte: Elaborado pelo autor

Na Tabela 2 o intervalo das classes foi de 0,75 minutos onde o menor valor analisado é 0,17 minutos e o maior 3,93 minutos. A classe que teve maior repetição foi a número 2 , com uma taxa de frequência de $35 \%$.

Tabela 2 - Tempo de Atendimento

\begin{tabular}{|c|c|c|c|c|}
\hline INTERVALO E & INTERVALO D & FREQ. ABS. & $\begin{array}{c}\text { FREQ. } \\
\text { RELATIVA }\end{array}$ & $\begin{array}{c}\text { FREQ. } \\
\text { ACUMULADA }\end{array}$ \\
\hline 0,92 & 1,67 & 22 & $35 \%$ & $65 \%$ \\
\hline 1,67 & 2,43 & 11 & $18 \%$ & $82 \%$ \\
\hline 0,17 & 0,92 & 18 & $29 \%$ & $29 \%$ \\
\hline 2,43 & 3,18 & 7 & $11 \%$ & $94 \%$ \\
\hline 3,18 & 3,93 & 4 & $6 \%$ & $100 \%$ \\
\hline
\end{tabular}

Fonte: Elaborado pelo autor

Conforme análise das tabelas 1 e 2, pode-se verificar que o tempo de atendimento varia bastante, com uma frequência acumulada, e que quase nunca é a mesma. Entende-se que, por motivos de quantidade de pagamento ou jogos, os intervalos $E$ e $D$ são consideralvelmente diferentes, e que ocorre a variação de frequência absoluda da amostra nestes intervalos.

\subsection{Dimensionamento de Médias}

Para que os números do dimensionamento sejam dados de forma mais correta, são usadas algumas fórmulas.

O ritmo, para verificar a chegada de clientes em razão a determinado tempo, o intervalo entre as chegadas, e a taxa e média de atendimento em geral. Para a média do desempenho do sistema, é necessário calcular a taxa de utilização do sistema, utilizando diversas outras fórmulas de probabilidade para um resultado mais exato.

O estudo foi realizado na Casa Lotérica, de Cataguases/MG, no bairro Centro, onde é visível o maior movimento, sendo um local de fácil coleta de dados. O horário escolhido foi o período do começo da tarde, por acreditar que, por ser um horário "livre" para a maioria da população que possui uma jornada de trabalho, ele aproveitariam para pagar suas contas. 
O sistema FIFO (First In, First Out) é caracterizado da seguinte forma = Primeiro que Entra, Primeiro que Sai) e, assim, após a realização do estudo e com a aplicação das fórmulas, foram encontrados os seguintes números.

Na tabela 3, pode-se verificar a chegada das pessoas para a fila em relação ao tempo que estava sendo cronometrado. A maior frequência de pessoas foi com a chegada de quatro no mesmo tempo. Assim, fluindo a fila.

Tabela 3: Tempo de Chegada

\begin{tabular}{|c|c|c|c|c|c|}
\hline Minuto & $\begin{array}{c}\text { Frequência } \\
\text { Observada }\end{array}$ & Minuto & $\begin{array}{c}\text { Frequência } \\
\text { Observada }\end{array}$ & Minuto & $\begin{array}{c}\text { Frequência } \\
\text { Observada }\end{array}$ \\
\hline 1 & 2 & 21 & 2 & 41 & 3 \\
\hline 2 & 1 & 22 & 4 & 42 & 1 \\
\hline 3 & 3 & 23 & 1 & 43 & 2 \\
\hline 4 & 4 & 24 & 3 & 44 & 1 \\
\hline 5 & 2 & 25 & 1 & 45 & 1 \\
\hline 6 & 2 & 26 & 4 & 46 & 2 \\
\hline 7 & 1 & 27 & 2 & 47 & 2 \\
\hline 8 & 3 & 28 & 3 & 48 & 4 \\
\hline 9 & 4 & 29 & 3 & 49 & 2 \\
\hline 10 & 1 & 30 & 1 & 50 & 1 \\
\hline 11 & 3 & 31 & 2 & 51 & 2 \\
\hline 12 & 1 & 32 & 2 & 52 & 3 \\
\hline 13 & 1 & 33 & 2 & 53 & 4 \\
\hline 14 & 3 & 34 & 1 & 54 & 2 \\
\hline 15 & 2 & 35 & 1 & 55 & 2 \\
\hline 16 & 2 & 36 & 4 & 56 & 2 \\
\hline 17 & 2 & 37 & 4 & 57 & 4 \\
\hline 18 & 2 & 38 & 1 & 58 & 1 \\
\hline 19 & 3 & 39 & 1 & 59 & 3 \\
\hline 20 & 1 & 40 & 3 & 60 & 2 \\
\hline
\end{tabular}

Fonte: Elaborado pelo autor

Conforme análise pode-se perceber que entre 10 a 40 minutos o número de chegadas aumentou. O maior número de clientes que chegaram, foram identificados nos minutos 4, 9 , 22, 26 36, 37, 48, 53 e 57. Consedera-se que se trata de clientes que possuem uma jornada de trabalho regular e que estão em seu intervalo para almoço, aproveitando para pagar contas e/ou realizar jogos.

$\mathrm{Na}$ tabela 4, é possível verificar a saída de pessoas da lotérica, e calcular o tempo de atendimento de cada cliente. 
Tabela 4: Tempo de Saída

\begin{tabular}{|c|c|c|c|c|c|}
\hline Minuto & $\begin{array}{c}\text { Frequência } \\
\text { Observada }\end{array}$ & Minuto & $\begin{array}{c}\text { Frequência } \\
\text { Observada }\end{array}$ & Minuto & $\begin{array}{c}\text { Frequência } \\
\text { Observada }\end{array}$ \\
\hline 1 & 1 & 21 & 1 & 41 & 1 \\
\hline 2 & 1 & 22 & 2 & 42 & 3 \\
\hline 3 & 1 & 23 & 3 & 43 & 2 \\
\hline 4 & 2 & 24 & 1 & 44 & 3 \\
\hline 5 & 3 & 25 & 2 & 45 & 1 \\
\hline 6 & 1 & 26 & 3 & 46 & 2 \\
\hline 7 & 2 & 27 & 4 & 47 & 2 \\
\hline 8 & 2 & 28 & 5 & 48 & 4 \\
\hline 9 & 3 & 29 & 2 & 49 & 3 \\
\hline 10 & 1 & 30 & 2 & 50 & 4 \\
\hline 11 & 4 & 31 & 1 & 51 & 2 \\
\hline 12 & 3 & 32 & 1 & 52 & 3 \\
\hline 13 & 3 & 33 & 1 & 53 & 2 \\
\hline 14 & 2 & 34 & 3 & 54 & 1 \\
\hline 15 & 3 & 35 & 1 & 55 & 1 \\
\hline 16 & 1 & 36 & 2 & 56 & 1 \\
\hline 17 & 5 & 37 & 2 & 57 & 4 \\
\hline 18 & 1 & 38 & 4 & 58 & 2 \\
\hline 19 & 2 & 39 & 3 & 59 & 2 \\
\hline 20 & 2 & 40 & 2 & 60 & 1 \\
\hline
\end{tabular}

Fonte: Elaborado pelo autor

Da mesma forma que a tabela 3, pode-se observar, analiticamente, as taxas de saída de clientes das filas na tabela 4. Após a realização do estudo e a aplicação das fórmulas, foram encontrados os números vizualisados nas tabelas 3 e 4 e foi demonstrado que é possível entender a estrutura do sistema e adequá-la à teoria das filas.

\section{Conclusão}

O estudo realizado apresenta forte potencial de continuidade, e possível ampliação dos eventos que foram observados, pois abrangeu uma Casa Lotérica da cidade de Cataguases MG, que possui um fluxo de atendimento pequeno, quando comparado às loterias de cidades maiores e com maior número de habitantes.

Após o levantamento e aplicabilidade, foi identificado o desempenho do sistema, fazendo a análise do modelo adotado por eles, entendendo que a Casa Lotérica poderia adotar o sistema de filas RR (Round-Robin), que acontece, resumidamente, da seguinte maneira: Atribui frações de tempo para cada processo em partes iguais e de forma circular, manipulando todos os processos sem prioridades. Em outras palavras, poderia existir para a Loteria, duas ou mais filas, dividindo em quantidade de serviços que as pessoas irão buscar. (Ex.: Fila 1: 1 a 5 contas. Fila 2: 5 ou mais contas), separando apenas às filas de Jogos e Preferencial, que ainda devem existir.

Assim, o serviço de uma das filas seria mais ágil, fazendo com que o fluxo fluísse ainda com mais praticidade. Diante do valor desde método para a análise eficiente e eficaz das filas, propõe-se ainda a realização de novos estudos com a aplicação desde método de teoria das filas, e ainda um comparativo do comportamento das filas de outras casas lotéricas existentes no município de Cataguases. 
O artigo indicou os pontos de melhoria e adaptação que deverá ser feito para que o mesmo possa aumentar sua rentabilidade, conquistando o cliente e consequentemente evitando a insatisfação dos consumidores já existentes.

\section{Referências Bibliográficas}

ANDRADE, E. L. de. Introdução à Pesquisa Operacional: métodos e modelos para análise de decisões. $4^{\mathrm{a}}$ ed- [Reimpr.]. Rio de Janeiro: LTC, 2011. 202 p.

APARECIDA, R.; História das loterias no Brasil, 05/Ago/2007; Disponível em: http://www.infoescola.com/historia/historia-das-loterias-no-brasil/. Acessado em: 20/jul, 2012.

CAMELO, G. R. COELHO, A, S. BORGES, R. M. SOUZA, R. M. Teoria das filas e da simulação aplicada ao embarque de minério de ferro e manganês no terminal marítimo de ponta da madeira. XXX Encontro Nacional De Engenharia De Produção, São Carlos, 2010.

Casa lotérica. In: Wikipédia, a enciclopédia livre. Disponível em: https://pt.wikipedia.org/wiki/Casa_lot\%C3\%A9rica. Acesso em: 01/nov, 2018.

DAL-FARRA, R. A. P.; LOPES, T. C.. Métodos Mistos de Pesquisa em Educação: pressupostos teóricos. Nuances: estudos sobre Educação, Presidente Prudente-SP, v. 24, n. 3, p. 67-80, set./dez. 2013. Disponível em: http://revista.fct.unesp.br/index.php/Nuances/article/ view/2698. Acesso em: 10 set. 2015.

DÁVALOS, P. B. Hidroquimica do Estuário do Rio Caravelas, Caravelas - BA / Pablo Bezzera Dávalos Natal, RN, 2012

DAVIS, M. M. Fundamentos da administração da produção. 3. ed. Porto Alegre: Bookman, 2001.

DE LIMA, B. P. A Teoria das Filas como ferramenta de apoio para Análise de uma empresa de lavarápido em Volta Redonda. Simpósio de Excelência e Gestão e Tecnologia, Resende, RJ, 28 out. 2015. Disponível em: https://www.aedb.br/seget/arquivos/artigos15/37422427.pdf. Acesso em: 31 out. 2018.

Estudo realizado sobre Teoria das Filas aplicado a uma Casa Lotérica no município de Marabá. 2015.p. Artigo (Bacharelado em Engenharia de Produção) - UEPA, [ENEGEP], 2015. 1. Disponível em: http://www.abepro.org.br/biblioteca/tn_stp_211_252_26707.pdf. Acesso em: 12 set. 2018.

FERREIRA, C.A. L.. Pesquisa Quantitativa e Qualitativa: Perspectivas para o campo da Educação. Revista Mosaico, Barcelona, p. 173-182, 28 out. 2015. Disponível em: https://www.aedb.br/seget/arquivos/artigos15/37422427.pdf. Acesso em: 31 out. 2018.

FOGLIATTI, M. \& MATTOS, N. Teoria de Filas. Ed. Interciência, 2007.

GROSS, D. SHORTLE, J. F. THOMPSON, J. M. HARRIS, C. M. Fundamentos da Teoria das Filas. New Jersey: John Wiley \& Sons, 2008.

HEIZER, J.; RENDER, B. Administração de Operações: Bens e Serviços. 5. ed. Rio de Janeiro: LTC, 2001. HILLIER, F. S.; LIEBERMAN, G. J. Introdução à pesquisa operacional. 8. ed. São Paulo: McGraw-Hill, 2006. JACOBS, F. R.; CHASE, R. B. Administração de Operações e da Cadeia de Suprimentos. 13. ed. São Paulo: McGraw-Hill, 2012.

LOVELOCK, C.; WRIGHT, L. Serviços: Marketing e Gestão. São Paulo: Saraiva, 2002.

PRADO, D. S. Teoria das Filas e da Simulação. Belo Horizonte, MG: Editora de Desenvolvimento Gerencial. Série Pesquisa Operacional, Vol. 2, 1999.

RENDER, B. Análise Quantitativa para Administração. 10 ed. Porto Alegre. Bookman. 2010.

RITZMAN, L. P.; KRAJEWSKI, L. J. Administração de Produção e Operações. São Paulo: Atlas, 2004

SABBADINI, F.; GONÇALVES, A. A.; OLIVEIRA, M. J. F. Gestão da Capacidade de Atendimento e Simulação Computacional para a Melhoria na Alocação de Recursos e no Nível de Serviço em Hospitais. Em: III SEGET - SIMPOSIO DE EXCELÊNCIA EM GESTÃO E TECNOLOGIA. 2006.

SILVA, M. B. Análise do Sistema de Filas de uma Casa Lotérica através da simulação e Teoria das Filas. XXXVI ENCONTRO NACIONAL DE ENGENHARIA DE PRODUCÃO, João Pessoa, 3 out. 2016. 